\title{
Chemical Constraints, Baryonic Mass and the Chemical Evolution of Low Surface Brightness Galaxies
}

\author{
M.G. Edmunds \\ Department of Physics and Astronomy, Cardiff University, P.O. Box \\ 913, Cardiff, CF2 3YB, U.K.
}

\begin{abstract}
A brief review of primordial helium and deuterium abundances suggests a baryonic mass density of $\Omega_{B} \approx 0.04-0.045$ (for $H_{0}=$ 70). This mass may be dominated by intergalactic gas in clusters and groups of galaxies. The observed low chemical abundances in evolved dwarf galaxies might suggest that outflow was the origin for such gas, and we make general suggestions for the interpretation of the data from the next generation of X-ray spectroscopic satellites. The effects of both outflow and inflow on the chemical evolution of galaxies is discussed, particularly in the context of low surface brightness galaxies, and we comment on their dust content.
\end{abstract}

\section{Baryonic Mass}

Pagel et al (1992) attempted to derive an accurate abundance for primordial helium, but neglected one systematic effect which may well have a significant influence. As pointed out by Izotov et al (1997) and Vilchez \& Iglesias-Páramo (1998) the neglect of underlying helium absorption lines of the stellar population in HII regions can lead to a slight underestimate of the strength of the helium emission lines, thus underestimating the helium abundance. A reasonable correction suggests a value of the primordial helium fraction closer to 0.24 than the Pagel et al value of 0.228 . The higher implied baryonic density is indicated in Figure 1. I think everyone aknowledges that $\mathrm{Y}$ must be less than 0.25 , and so the helium abundances come into better agreement with the deuterium data. For the latter, Tosi et al (1998) give a lower limit on astration destruction from detailed numerical chemical evolution of the Galaxy which agrees quite well with analytical limits (Edmunds 1994), and the observed Galactic deuterium abundance provides an upper limit. Tytler's qso absorption-line measurements lie nicely in between (Tytler, Fan \& Burles 1996, scaled to $H_{0}=70$; a slightly lower value of 0.039 may be indicated by more recent work - Burles \& Tytler 1998). Looking at Figure 1 suggests that a value of $\Omega_{B}$ between 0.04 and 0.05 would fit the He and D data - I hesitate to include lithium because of the difficulties associated with its interpretation (but see Pagel in this volume or his book 1997). We are assuming $H_{o}=70$, but the value of say $0.040-0.045$ for $\Omega_{B}$ would be multiplied by $\left(H_{\circ} / 70\right)^{-2}$ for other values of $H_{0}$.

In the lower part of Figure 1, we give some estimates of "observed" baryonic mass. It would be better to say "inferred" rather than "observed", since the argument is rather indirect and must proceed through various assumptions about 


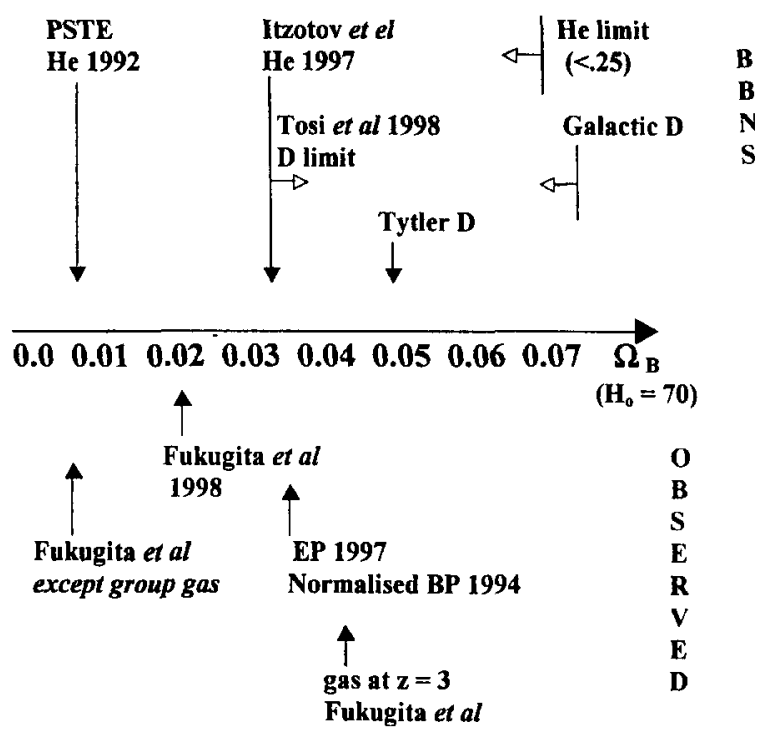

Figure 1. Baryonic Mass Estimates

mass-to-light ratios and so on. The lowest point here is from a useful compilation by Fukugita, Hogan \& Peebles (1998). This low point includes intergalactic gas in clusters of galaxies (where it dominates the baryonic mass, comprising perhaps three times the total baryonic mass actually in galaxies) but neglecting any intergalactic gas in groups. What Fukugita et al point out is that if groups had an intergalactic gas fraction similar to that in clusters it would dominate the baryonic mass, and push the point to the right as shown. Now by stretching mass-to-light ratios etc, it is possible to push the inferred galaxies' mass further to the right, as indicated by the point EP 1997 - which represents using the galaxy numbers etc detailed in Edmunds \& Phillipps (1997) with the extreme mass-to-light ratios of Bristow \& Phillipps (1994). This is probably a bit of an overestimate for the galaxy mass, and it includes cluster gas, but not group gas. However, it is evident that it is almost reaching the $\Omega_{B}$ implied by the big-bang nucleosynthesis results, and could certainly do so with judicious addition of gas in groups. A final point is to note the (very uncertain) baryonic mass of gas at redshift 3 , as implied from qso absorption clouds - and presumably representing what will subsequently become galaxies or remain as intergalactic gas. So we see that there is a sort of consistent picture with $\Omega_{B} \approx 0.04-0.045$, but that it probably implies the existence of considerable intergalactic gas, much of which could be in groups. This group gas, being in a smaller potential well and rather cooler than cluster gas, might well have escaped significant detection so far - but be a prime target for the next generation of $\mathrm{X}$-ray telescopes. We now speculate on its origin and composition. 


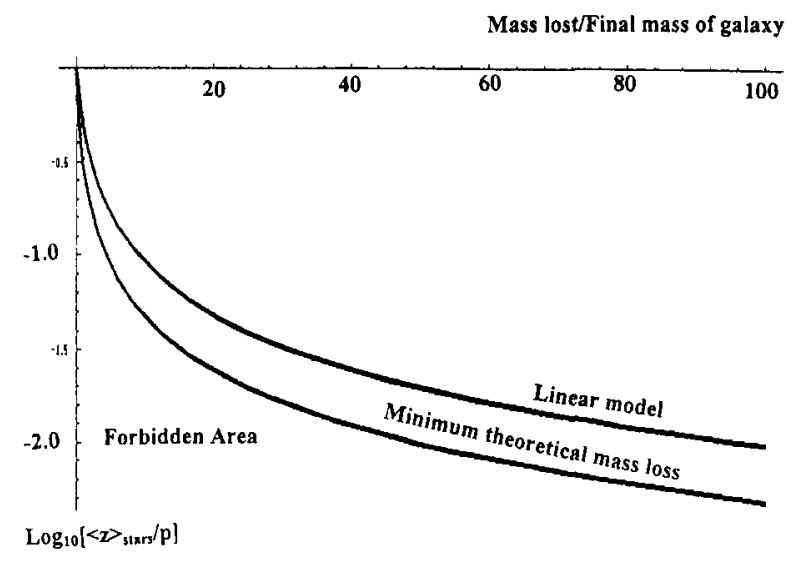

Figure 2. Inevitable Mass Loss for Low-Metallicity Galaxies

\section{Galactic Mass Loss and Intergalactic Gas}

The possibility of global mass loss influencing the chemical evolution of galaxies, and enriching the intergalactic medium - particularly in clusters - has been discussed for many years. What motivates my comments is the realisation that it is easy to give (Edmunds 1999) a lower limit on the gas mass flowing out of galaxies, under certain (fairly standard and reasonable) conditions. The conditions are the assumptions usually made in analytic chemical evolution modelling - instantaneous recycling, that the gas in the galactic system is well-mixed (i.e. any outflow is not preferentially metal-enhances by, for example, direct outflow of supernova products), and that the intrinsic chemical yield (mainly constrained by the initial mass function for star formation) does not vary. Then a system which has lost mass, so that only a fraction $M$ of its initial mass remains when all the gas has been converted into stars, must have a mass-weighted stellar metallicity equal to or greater than $\mathrm{p}[1-(1-1 / \mathrm{M}) \operatorname{Ln}(1-\mathrm{M})]$. Thus if we see a gas-exhausted system with a low mean stellar metallicity $\langle z\rangle_{\text {stars }}$ it must have undergone considerable mass loss - unless one or more of the assumptions is invalid. The implied gas loss is shown in Figure 2, which is a plot of the above equation. It also shows the even higher mass loss implied for the popular "linear" outflow model in which the outflow rate is simply proportional to the star formation rate. Now suppose (following references in Phillipps \& Edmunds 1996) that we assume a metallicity-luminosity relation for galaxies - for which there is reasonable observational evidence - of the form $\mathrm{z}(\mathrm{L}) \alpha L^{k}$ where $\mathrm{k} \sim 0.5$ (or perhaps a bit shallower: van Zee, Haynes \& Salzer's 1997 Figure 1 suggests $\mathrm{z} \sim L^{0.36}$ for gas-rich, lsb dwarfs. Here we are really talking about gas-poor, evolved systems). Also assume a luminosity function of the form $\mathrm{N}(\mathrm{L}) \alpha L^{\alpha}$, at least for moderate and low mass galaxies, and a constant mass-to-light ratio. The metallicity-luminosity relation implies (via the mass-loss equation) the minimum mass that a galaxy of present luminosity L must have lost, and one can integrate over the luminosity function to find the ratio (minimum mass lost from galaxies $) /($ mass now in galaxies). A little numerical integration, or approxima- 


\section{Metals in groups and clusters (Detailed X-ray analysis awaited!)}

\begin{tabular}{l|l|l} 
& $\begin{array}{l}\text { High } \\
\text { metals }\end{array}$ & $\begin{array}{l}\text { Low } \\
\text { metals }\end{array}$ \\
\hline Little gas & $\begin{array}{l}\text { Metal- } \\
\text { enhanced } \\
\text { outflow }\end{array}$ & $\begin{array}{l}\text { Outflow not } \\
\text { universally } \\
\text { important }\end{array}$ \\
\hline Much gas & $\begin{array}{l}\text { Current } \\
\text { yields very } \\
\text { wrong and } \\
\text { outflow very } \\
\text { important }\end{array}$ & $\begin{array}{l}\text { Either } \\
\text { (i) outflow } \\
\text { important- } \\
\text { if } z \sim \text { as } \\
\text { expected } \\
\text { or } \\
\text { (ii) if } z \text { very } \\
\text { low, outflow } \\
\text { not } \\
\text { important }\end{array}$
\end{tabular}

Figure 3. "Outflow" Interpretations of Intergalactic Gas

tion, soon shows that this ratio can be of order three, if we take an $L_{*}$ galaxy to have lost no mass (and hence have $\langle z\rangle / p=1$ ) and integrate down to, say, $0.001 L_{*}$. So again a picture can arise in which the intra-cluster and intra-group gas has been through the galaxies, but now dominates the baryonic mass by the right amount to give consistency with the baryonic density implied by big-bang nucleosynthesis. Rough estimates can be made of the metallicity of the gas (for details see Edmunds 1999), and it must be low-ish, perhaps $1 / 5$ to $1 / 4$ solar, if we stick to the standard assumption of the yield $p$ being around solar. Of course, this and our other assumptions could be wrong - in particular, metal-enriched outflow might be occuring. The outflowing gas may mix with unenriched gas that never went through galaxies, although we might then start predicting too much intergalactic gas if there is significant outflow! Here abundances would be very low. We can make a rough table (Figure 3 ) of what we would conclude from future X-ray observations if they show little gas or lots, and its metallicity.

\section{Chemical Evolution of LSB Galaxies}

Is the chemical evolution of Low Surface Brightness (LSB) galaxies much the same as for high surface brightness (HSB) galaxies? As an initial try at answering this question, Figure 4 is a plot of gas metallicity versus gas fraction for 


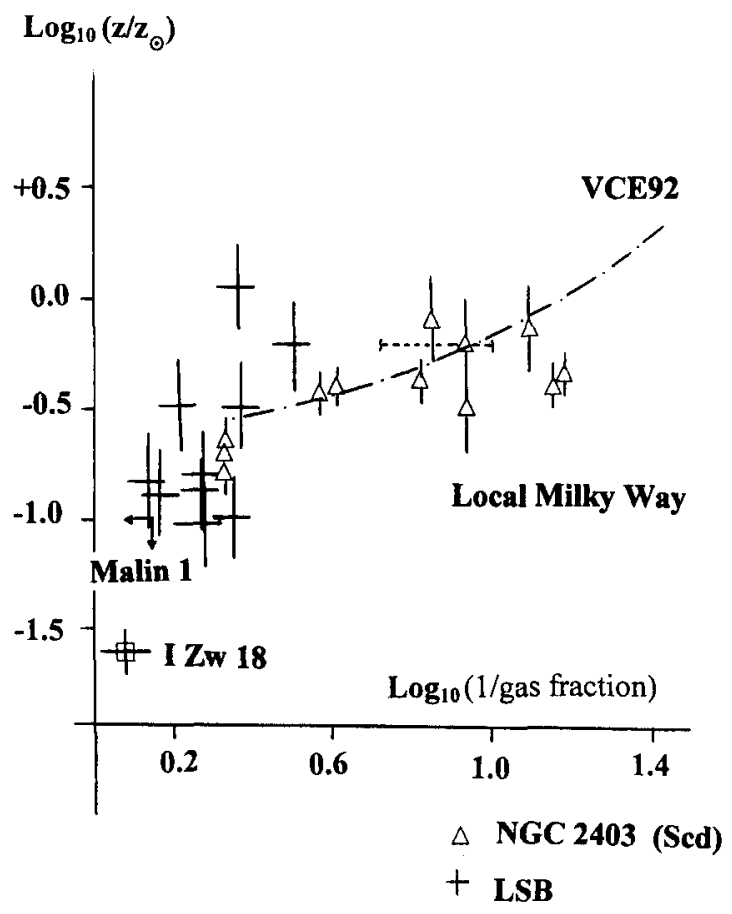

Figure 4. Gas Abundances versus Gas Fraction

both LSBs and HSBs. On such a diagram the simple "closed box" model for chemical evolution would have $\mathrm{z} / \mathrm{p}=\operatorname{Ln}(1 /$ gas fraction), and it is possible to show (Köppen \& Edmunds 1999, Edmunds 1999) that nearly any type of inflow - particularly time-decreasing inflow - does not cause a major displacement from this line. To move away significantly requires either outflow or rather sudden, rapid accretion onto a well-evolved system. It is quite easy (within the errors) to pass a single simple model curve through most of the points on Figure 3 so there is no evidence here that $L S B$ galaxies are intrinsically different in their chemical evolution from HSB galaxies. Van Zee, Haynes \& Salzer (1997) reach a similar conclusion. On the diagram the crosses are LSB data mainly taken from McGaugh (1994), McGaugh \& de Blok (1997). The Malin I point is not the nucleus whose spectrum does not look particularly metal poor, the I Zw 18 abundance is from Pagel et al 1992 with gas fraction from data in van Zee et al 1998 with star mass from luminosity rather than dynamics. The triangles are the Scd spiral NGC 2403 from Garnett et al (1997). The dash-dot line is a mean reation for spiral disks from Vila-Costas \& Edmunds (1992), and the local Milky Way (i.e. solar neighbourhood) is marked as a short horizontal dashed line.

Since LSB galaxies apparently show less obvious spiral structure than HSB galaxies, one might wonder if they might not show similar radial abundance gradients - since the organisation of star formation by spiral structure is a good candidate mechanism for gradient generation (e.g. Wyse \& Silk 1989). Both spiral structure and abundance gradients seem to die in galaxies fainter than $M_{V} \sim-17$ 
(Edmunds \& Roy 1993), and it would be fascinating to know if LSB galaxies with absolute magnitudes brighter than this also show a lack of both attributes. Thijs van der Hulst and his colleagues have started looking at this problem (e.g. de Blok \& van der Hulst 1998), but more data is needed before any conclusions can be drawn.

There is, of course, the apparent observational correlation of abundance with surface brightness (e.g. Ryder 1995, and Skillman in this volume) - but its true origin is still unclear, although self-regulation of star formation is a possibility. It is important to make sure that any observed correlation is not simply due to gas fraction (i.e. a lower star formation rate and consequently less heavy elements) rather that some special coupling to other local conditions. I do wonder if some of the "second parameter" behaviour in the luminosity/metallicity relation mentioned in Evan Skillman's paper (this volume) might simply arise from different gas fractions.

\section{Dust}

I hesitate to say much about dust in LSB galaxies, except to emphasise that the formation/destruction cycle for dust is probably not yet understood in any type of galaxy - although there are indications of progress (Dwek 1998, Tielens 1998). Steve Eales (Edmunds \& Eales 1998) and I have tried to set some very elementary limits on dust masses in galaxies from simple abundance constraints, assuming that an approximately fixed fraction of interstellar metals condense into/onto dust. If metal abundance is really the controlling factor, then LSBs should be no different in dust content (for a given gas fraction) than HSBs. If it is other mechanisms that dominate - e.g. destruction rate through supernova explosions. growth rate of dust grain mantles (which is where the bulk of mass lies) in dense clouds - then LSBs might well have different dust content, if the supernova rate or interstellar cloud mass/number/density spectrum is significantly different. We are beginning work to try and understand the dust creation-destruction cycle better, and any (even qualitative) predictions lie only in the future - but comparisons between LSB and HSB galaxies will provide a useful testbed for ideas.

\section{Final Thought}

One of the participants at this meeting rather nicely described a low surface brightness galaxies as "a big baryonic gas bag" - I fear that after this talk, he'll apply the description to me....

\section{References}

Bristow, P.D. \& Phillipps, S. 1994, MNRAS, 267, 13

Burles, S. \& Tytler, D. 1998, ApJ, 507, 732

de Blok, W.J.G. \& van der Hulst, J.M. 1998, A\&A, 335, 421

Dwek, E. 1998, ApJ, 501, 643 
Edmunds, M.G. 1994, MNRAS, 270, L37

Edmunds, M.G. 1999, MNRAS in preparation

Edmunds \& Eales, S.A. 1998, MNRAS, 299, L29

Edmunds, M.G. \& Phillipps, S. 1997, MNRAS, 292, 733

Edmunds, M.G. \& Roy, J-R., 1993, MNRAS, 261. L17

Fukugita, M., Hogan, C.J. \& Peebles, P.J.E. 1998, ApJ, 503, 518

Garnett, D.R., Shields, G.A., Skillman, E.D., Sagan, S.P. \& Dufour, R.J. 1997, ApJ, 489, 63

Izotov, Y.I., Thuan, T.X. \& Lipovetski, V.A. 1997, ApJS, 108, 1

Köppen, J. \& Edmunds, M.G. 1999, MNRAS, in press

McGaugh, S.S. 1994, ApJ, 426, 135

McGaugh, S.S. \& de Blok, W.J.G. 1997, 481, 689

Pagel, B.E.J. 1997 Nucleosynthesis and the Chemical Evolution of Galaxies, Cambridge Univesity Press.

Pagel, B.E.J., Edmunds, M.G., Simonson, E.A.\& Terlevich, R.J. 1992, MNRAS, 255,325

Phillipps, S. \& Edmunds, M.G. 1996, MNRAS, 281, 362

Ryder, S.D. 1995, ApJ, 444, 610

Tielens, A.G.G.M. 1998, ApJ, 499, 267

Tosi, M., Steigman, G., Matteucci, F. \& Chiappini, C. 1998, ApJ, 498, 226

Tytler, D., Fan, X.M. \& Burles, S. 1996, Nature, 381, 207

van Zee, L., Haynes, M.P. \& Salzer, J.J. 1997, AJ, 114, 2497

van Zee, L., Westpfahl, D., Haynes, M.P. \& Salzer, J.J. 1998, AJ, 115, 1000

Vila-Costas, M.B. \& Edmunds, M.G. 1982, MNRAS 259, 121

Vilchez, J.M. \& Ingesias-Páramo, J. 1998, in Abundance Profiles: Diagnostic Tools for Galaxy History, ASP Conference Series, Vol 147 eds D. Friedli, M.G. Edmunds, C. Robert $\&$ L. Drissen 120

Wyse, R. \& Silk, J. 1989, ApJ, 339, 700 